\title{
AÇÕES DE CUIDADORES NA PREVENÇÃO E TRATAMENTO DE LESÕES DE PELE NO IDOSO
}

\section{CAREGIVER ACTIONS IN THE PREVENTION AND TREATMENT OF SKIN LE- SIONS IN THE ELDERLY}

\author{
Juliana Balbinot Reis Girondi ${ }^{1} *$ Luciara Fabiane Sebold $^{2} *$ Andressa Maria Gomes $^{3}$ * \\ Daniela Soldera $^{4} *$ Bettina Heidenreich Silva $^{5} *$ Maria Eduarda Alves Ferreira $^{6}$
}

\section{RESUMO}

Os idosos são mais susceptíveis a agravos como as Lesões por Pressão e Lesões por Fricção. Objetivo: identificar as ações e cuidados com a pele dos idosos desenvolvidos por seus cuidadores. Método: Estudo qualitativo do tipo exploratório-descritivo desenvolvido nos meses de agosto e setembro de 2018, em uma comunidade de Florianópolis/Santa Catarina. A coleta de dados ocorreu através de entrevista semiestruturada, gravada e transcrita, com 20 cuidadores da referida comunidade; submetidas a análise temática. Resultados: Emergiu duas categorias temáticas: Cuidados gerais com a pele do idoso e Principais cuidados com lesões de pele no idoso. Conclusão: Foi possível identificar o conhecimento e as principais ações de prevenção e tratamento que os cuidadores empregam no cuidado de pele do idoso, sendo as mais destacadas hidratação, higiene e mudança de decúbito.

Palavras-chave: Enfermagem; Fricção; Lesão por pressão; Pele; Saúde do Idoso.

\section{ABSTRACT}

The elderly are more suscptible to injures such as Pressure Injuries and friction injuries. Objective: identify the actions and skin of the elderly developed by their caregivers. Method: Qualitative exploratory-descriptive study developed in the months of august and september 2018, in a community in Florianópolis/Santa Catarina. Data collection took place through semi-structures interviews, recorded and transcribed, with 20 caregivers from that community; subject to thematic analysis. Results: Two thematic categories emerged: General skin care for the elderly and Main care for skin injuries in the elderly. Conclusion: It was possible to identify the knowledge and the main prevention and treatment actions that caregivers employ in skin care for the elderly, the most prominent being hydration, hygiene and change of position.

Keywords: Nursing; Friction; Pressure Injury; Skin; Elderly Health.

\footnotetext{
${ }^{1}$ Dra. Docente Departamento de Enfermagem da Universidade Federal de Santa Catarina. Orcid: https://orcid.org/00000003-0271-259X

2 Dra. Docente Departamento de Enfermagem da Universidade Federal de Santa Catarina - UFSC. Orcid: 0000.0002 .5023 .9058

${ }^{3}$ Enfermeira. Orcid: 0000.0002.0641.9588

${ }^{4}$ Doutoranda Profissional em Gestão do Cuidado em Enfermagem - Universidade Federal de Santa Catarina - UFSC. Orcid: https://orcid.org/0000-0002-6962-1556

5 Acadêmica de Enfermagem. Universidade Federal de Santa Catarina. Orcid: https://orcid.org/0000-0002-7623-7085

${ }^{6}$ Acadêmica de Enfermagem. Universidade Federal de Santa Catarina. Orcid: 0000.0002.2593.1659
} 


\section{INTRODUÇÃO}

Apesar do aumento da expectativa de vida, a população está envelhecendo de forma acelerada e em condições socioeconômicas e culturais desfavoráveis ${ }^{(1)}$. No processo de envelhecimento ocorrem modificações no organismo como alterações morfológicas, funcionais e bioquímicas, o que aumenta a susceptibilidade das doenças, principalmente as crônico-degenerativas, que podem provocar limitações e dependências, bem como situações de vulnerabilidade, fragilidades, incapacidades e as mais variadas necessidades de saúde do idoso ${ }^{(2)}$.Como parte do processo de envelhecimento há a diminuição do turgor cutâneo, diminuição da sensibilidade, da acuidade auditiva e visual, perda do equilíbrio, como também são acometidos pelas Doenças Crônicas Não Transmissíveis, dentre elas a hipertensão, diabetes, depressão, osteoporose, Parkinson, Alzheimer, que intensificam o risco de quedas e fraturas, que podem ocasionar a restrição ou perda da mobilidade, deixando-os mais susceptíveis a agravos como as Lesões por Pressão (LP) e Lesões por Fricção (LF).

As LP e LF são lesões que causam impacto significativo para os pacientes, familiares e sistema de saúde, por serem recorrentes, incapacitantes e repercutirem de forma severa na qualidade de vida desses ao causarem dor e sofrimento; as quais poderiam ser prevenidas ${ }^{(3)}$.

As LP é definida como uma área de necrose celular que tende a se desenvolver quando o tecido mole é comprimido entre uma https://doi.org/10.31011/reaid-2021-v.95-n.34-art.998 Rev Enferm Atual In Derme v. 95, n. 34, 2021 e-021060

proeminência óssea e uma superfície dura por um período prolongado de tempo. São causadas por fatores intrínsecos e extrínsecos ao paciente. A pressão, o cisalhamento, a fricção e a umidade em um paciente acamado são fatores extrínsecos que podem levar ao aparecimento destas lesões ${ }^{(4)}$.

A LF, que vem do termo em Inglês Skin tears, são feridas traumáticas rasas, que ocorrem principalmente nas extremidades do corpo, resultante de fricção ou de uma combinação de fricção e cisalhamento, levando à separação da epiderme da derme (ferida de espessura parcial) ou separando totalmente a epiderme e a derme das estruturas subjacentes (ferida de espessura total). São encaradas como inerentes à idade, induzindo à sensação de uma condição irrelevante, porém acarretam dor e infectam facilmente. A produção de exsudato seroso, especialmente nas primeiras 24 horas, torna as LF majoritariamente úmidas. Muito embora os números brasileiros atuais deste tipo de lesão permaneçam ocultos, sua prevalência é maior do que as LP ${ }^{(5)}$.

Estas lesões de pele, por serem dolorosas e incapacitantes, fazem com que os idosos necessitem de auxílio de uma rede de apoio, e ainda, quando o familiar não tem capacidade física ou emocional, uma pessoa da comunidade que foi adquirindo experiência por meio do cuidado à pessoas doentes, faz disso uma ocupação informal (2).

Conhecer a dinâmica do cuidado familiar prestado ao idoso no cuidado da sua pele é essencial para subsidiar a equipe de 
saúde, em especial a Enfermagem. Dessa forma poderemos trabalhar com as reais necessidades e especificidades, buscando instrumentalizar esse cuidadores para a adoção de práticas seguras e eficazes, pautadas nas melhores evidências científicas em relação ao cuidado da pele do idoso. Frente a essas constatações emergiu a pergunta de pesquisa: "Quais ações e cuidados com a pele dos idosos são desenvolvidos por seus cuidadores?"

Doravante, o estudo objetivou identificar as ações e cuidados com a pele dos idosos desenvolvidos por seus cuidadores.

\section{MÉTODO}

Pesquisa qualitativa, do tipo exploratório-descritivo, realizada no período de agosto e setembro de 2018 numa unidade básica de saúde localizada no município de Florianópolis, Santa Catarina. Essa unidade abrange um contigente expressivo de idosos (664 idosos), que representa 12\% da população municipal ${ }^{(6)}$.

Participaram do estudo 20 cuidadores de idosos residentes e cadastrados pelas equipes de saúde da família, na área adscrita da comunidade. Foram considerados como critérios de inclusão: ser familiar, acompanhante/cuidador de idosos com idade igual ou superior a 60 anos e ter acima de 18 anos; e critérios de exclusão: não estar disponível para participar da pesquisa no período da coleta de dados.

Para a coleta dos dados foi utilizada entrevista semiestruturada. Os coletadores de dados foram treinados, mediante aplicação de https://doi.org/10.31011/reaid-2021-v.95-n.34-art.998 Rev Enferm Atual In Derme v. 95, n. 34, 2021 e-021060 roteiro semiestruturado, sendo as principais: uma aluna da graduação em enfermagem e sua orientadora. Todas as entrevistas foram gravadas em formato de áudio e posteriormente transcritas para facilitar a análise dos dados. Os dados foram analisados conforme Análise Temática de Minayo.

O estudo está vinculado ao macroprojeto de pesquisa intitulado: "Estratificação de risco e intervenções de enfermagem no diagnóstico, prevenção e tratamento de skin tears e úlceras por pressão em idosos", aprovado sob parecer n ${ }^{\circ} 2.390 .948$ e CAAE 74769317.5.1001.0121, financiado pela Coordenação de Aperfeiçoamento de Pessoal de Nível Superior ( CAPES).

\section{RESULTADOS}

Os participantes da pesquisa foram 20 cuidadores, sendo 13 familiares e sete cuidadores com qualificação profissional, todos do sexo feminino, tendo idades entre 32 anos e 65 anos, com média de 53 anos. Da análise dos dados emergiram duas categorias temáticas.

\section{Cuidados Gerais com a pele do idoso}

Nesta categoria os cuidadores apresentaram seus conhecimentos acerca dos fatores de risco, assim como os cuidados gerais que devem ser adotados no cuidado da pele do idoso, geralmente obtidos por senso comum. As sete entrevistadas identificaram como risco de desenvolvimento de lesões a manutenção do idoso na mesma posição por muito tempo, porém sem especificar este tempo. Doravante, 
compreendem a pele do idoso como sendo mais frágil, adotando como cuidado o reposicionamento no leito.

A pele do idoso é uma pele mais velha, mais frágil, fica mais na mesma posição, sendo importante sempre fazer essa troca... (C3)

Outros cuidadores ressaltaram a questão da higiene corporal como sendo um importante fator de risco se não executada adequadamente e periodicamente. Para mantê-la, destacam como cuidados: banho diário, uso de sabonete neutro, troca de roupa de cama, troca de fralda, uso de absorventes em casos de incontinência urinaria, cuidados com controle de evacuações, uso de AGE como hidratante diário para a pele.

Segundo orientação que eu tenho é importante deixar os lençóis bem esticados, para não machucar a pele do idoso $(\mathrm{C} 1)$

A gente dá uns absorventes para ela, ela tem incontinência, acho que esse é o único cuidado que faço $(\mathrm{C} 11)$

O sol foi identificado como um fator de risco para desenvolvimento de lesão na pele do idoso. Embora em menor representatividade, os cuidadores apontam a importância do uso de protetor solar e da necessidade de tomar banho de sol nos horários adequados.

Sempre vamos fazer umas caminhadas no final da tarde, mas sempre tenho que lembra ela de passar protetor $(\mathrm{C} 9)$
Por ter tido câncer de pele, cuido mais disso até sou chata as vezes, com o protetor $(\mathrm{C} 17)$

Destaque está para a associação que os cuidadores fizeram sobre as Doenças Crônicas Não Degenerativas, como Diabetes Mellitus, em relação a dificuldade de cicatrização, representando um fator de risco para o desenvolvimento de lesões.

O da mãe é a diabetes, se ela se corta, bate, machuca, demora para cicatrizar $(\mathrm{C} 15)$

Os cuidadores identificaram como outros fatores de risco: traumas ocasionados por batidas em móveis, friccionar as pernas em objetos, dobras de lençóis, a presença de varizes e a realização de exercícios repetitivos em pacientes acamados. Outros cuidados desenvolvidos estão relacionados a alimentação, hidratação oral, uso de cama hospitalar e de dispositivos como colchões d'água e caixa de ovo.

Estamos sempre cuidando para ela não bater nas coisas, não arrastar a perna em nada (C10)

Quando ele ficou doente conseguimos uma cama hospitalar..., também tínhamos um colchão de água e um outro tipo caixa de ovo em cima (C18)

Apesar da maioria dos cuidadores possuir conhecimentos adequados sobre o cuidado a pele do idoso, uma importante parcela relataram não ter conhecimento sobre 
esse aspecto delegando ao idoso responsabilidade e condutas sobre o seu autocuidado.

A fim de prevenir lesões os cuidadores desenvolveram ações e cuidados preventivos, quais sejam: reposicionamento e alteração de decúbito, hidratação cutânea, higiene corporal, uso de protetor solar e manutenção de lençóis sem dobras. Destaca-se ainda a importância de usar calçados adequados, uso de meias compressivas, estímulo à deambulação e promoção de ambiente acessível. Um respondente aponta o uso de placa de hidrocolóide como cuidado preventivo.

Quando ela fica muito na cama, gosto de chamar ela para dar uma volta, vamos caminhar na praia... (C5)

Cuido sempre com a casa para ela não bater em nada, não ter em nada em que bater... (C9)

Quando ele tava mais acamado usávamos uma plaquinha de hidrocolóide. (C18)

\section{Os cuidados com lesões na pele do}

\section{idoso}

Nessa categoria os cuidadores apontam quais cuidados eles desenvolvem com lesões de pele já instauradas e os profissionais que mais o auxiliam neste processo de cuidar. Os cuidadores destacaram como principal adjuvante neste cuidado a procura pela equipe de saúde, sendo que citaram os principais profissionais sendo, os enfermeiros, os médicos e técnicas de enfermagem. Como também citaram, se tivessem alguma dúvida a equipe de saúde a esclareceria, não sentindo assim, necessidade de informações por outras vias.

Se ele tem algo, a gente já vem para o posto, o pessoal ajuda muito $(\mathrm{C} 4)$

Qualquer coisa já corremos para o posto, na idade dela temos que cuidar... umas das meninas sempre nos auxilia nessas horas (C11)

Alguns dos cuidados no tratamento das lesões de pele citados foram: a utilização do Àcido Graxo Essencial, a limpeza com solução fisiológica, o uso de placa hidrocolóide (um respondente), a mudança de decúbito (um respondente), o cuidado na mobilização (um respondente) e o uso de pomada de colagenase (um respondente). Sendo que um cuidador apontou que realiza a massagem nas áreas de lesão.

$\mathrm{Na}$ lesão dele eu limpo com soro, depois uso colagenase na área de necrose e AGE em volta (C2)

Ele nunca teve nada desse tipo, nós cuidamos bastante, o geriatra dele até ficou impressionado dele não ter nada (C3)

\section{DISCUSSÃO}

Os fatores de risco para o desenvolvimento de LP são: a sensibilidade reduzida, alterações cutâneas, imobilidade prolongada ou idade avançada. Está associada 
principalmente às mudanças degenerativas da pele e/ou tecido subcutâneo exposto às forças de pressão e cisalhamento; em decorrência da pressão sobre a proeminência óssea, a qual prejudica a circulação sanguínea, favorecendo dessa forma a morte celular e o consequente aparecimento da lesões em locais de maior risco, como as regiões occipital, escapular, cotovelo, sacral, ísqueo, trocanter, crista ilíaca, joelho, maléolo e calcâneo ${ }^{(7)}$.

$\mathrm{Na}$ pesquisa verificou-se que os cuidadores possuem essa percepção, uma vez que sinalizam que a pele do idoso tem maior fragilidade, $\log$ apresenta maior vulnerabilidade para o desenvolvimento lesões.

$\mathrm{Na}$ categoria cuidados gerais com a pele do idoso, os cuidadores destacam vários aspectos importantes e fundamentais para a manutenção da integridade cutânea, considerando especialmente os fatores de risco associados. Uma das maiores preocupações diz respeito à questão do posicionamento e reposicionamento, no entanto, os participantes não especificam a periodicidade da alternância de decúbito, nem tampouco sobre a angulação adequada ao reposicionar esse paciente. Estudiosos apontam a importância de se implementar medidas preventivas como elevar a cabeceira da cama no máximo $30^{\circ}$, uso de camas e colchões especiais para redistribuir pressão, utilizar forro móvel para mover ou transferir pacientes acamados, mudança de decúbito e troca regular de fraldas $^{(8)}$. A alternância de decúbito deverá ser realizada preferencialmente de duas em duas horas, para evitar a pressão prolongada e também a redução da irrigação sanguínea local.

Além da diminuição da mobilidade dos idosos, há o uso de fraldas, que aumenta o risco de LP devido à predisposição a um processo de maceração da pele que, se não controlada, leva a uma redução na força tensiva, tornando-a susceptível à compressão, fricção e cisalhamento, decorrentes da exposição à umidade por períodos prolongados. Isto reforça a necessidade de conscientização dos cuidadores/familiares a promover cuidados adequados de higiene, como também estimular o autocuidado do idoso $^{(8)}$.

Outro aspecto trazido pelos cuidadores com propriedade foi em relação à higiene e hidratação. Destaca-se a importância da hidratação da pele e do uso de sabonete adequado para prevenir o ressecamento $\mathrm{e}$ melhorar a elasticidade, evitando assim, as fissuras na pele. Os cuidadores de forma geral reafirmam a importância destes cuidados ressaltando os relacionados à higiene corporal, uso de sabonete neutro, hidratação corporal cutânea diária e controle de eliminações vesico-intestinais ${ }^{(9)}$. O momento da higiene do idoso, é tanto importante para remoção das sujidades, como para observar a presença de lesões de pele. Dada as especificidade da pele do idoso, o recomendado atualmente é a utilização de um sabonete com ph ligeiramente acidificado ( $\mathrm{ph}$ entre 4,5 a 5,5). $\mathrm{O}$ uso do hidratante é recomendado após o banho especialmente quando a pele estiver seca, fato este não descrito pelos participantes de nosso https://doi.org/10.31011/reaid-2021-v.95-n.34-art.998 Rev Enferm Atual In Derme v. 95, n. 34, 2021 e-021060 
estudo. Embora o produto mais apropriado ainda não tenha sido preconizado, existem resultados promissores em relação aos Ácidos Graxos Essenciais (AGE) ${ }^{(10)}$.

A hidratação cutânea de superfície pode ocorrer por oclusão ou por umectação (hidrófila). Na primeira, a evaporação da água é reduzida, ou seja, são substâncias lipídicas ou emolientes, que formam uma película na superfície da pele, conhecidas como emulsões à base de óleos vegetais, como o AGE. Na segunda, as substâncias são umectantes (hidrofílicas), assim, a aplicação de cremes ou vaselina líquida diariamente é mais favorável a esse processo ${ }^{(11)}$. Foi citado como meio preventivo o uso de placa hidrocolóide, apesar de sua indicação principal ser para tratamento de lesões, também pode ser utilizada como meio de diminuir a pressão óssea e o cisalhamento $^{(12)}$.

Ainda sobre os cuidados gerais com a pele do idoso, um respondente apontou a realização de massagem de conforto em região de proeminências ósseas. Cabe destacar que a massagem é contraindicada na presença de inflamação aguda e onde existe a possibilidade de haver vasos sanguíneos danificados ou pele frágil. Dessa forma, a massagem não deverá ser recomendada como uma estratégia de prevenção de lesões por pressão, como relatado por um participante da pesquisa ${ }^{(13)}$.

Outro cuidado geral com a pele diz respeito à exposição solar adequada, a qual pode ser benéfica para saúde do idoso e sua pele, como relatado pelos cuidadores. Para tal, o banho de sol deve acontecer https://doi.org/10.31011/reaid-2021-v.95-n.34-art.998 Rev Enferm Atual In Derme v. 95, n. 34, 2021 e-021060 preferencialmente, antes da 10 horas e depois das 16 horas, por um período de 10 a 20 minutos, afim de otimizar a síntese de vitamina D. Esta vitamina pode se encontrar diminuída no organismo do idoso por alguns fatores, como: exposição menor ao sol, redução da capacidade de produção cutânea de vitamina $\mathrm{D}$, alimentação inadequada, diminuição de sua absorção pelo trato gastrintestinal, comprometimento renal e utilização de múltiplas drogas que interferem na absorção/metabolização da vitamina D. No entanto, ela é fundamental para a manutenção do tecido ósseo, do sistema imunológico, reforçando-se sempre o uso do protetor solar em qualquer horário do dia ${ }^{(14)}$.

Alguns cuidadores colocaram a questão das DCNT, como a DM, como fator de risco para o desenvolvimento de lesões, associando-a ao processo de cicatrização. No paciente diabético, ocorre dificuldade de cicatrização das feridas, devido ao comprometimento da perfusão sanguínea, impedindo o fornecimento adequado de oxigênio, nutrientes e antibióticos, principalmente nos membros inferiores. Isso leva à desorganização dos estágios iniciais de reparo, ocasionando atraso no processo de regeneração tecidual ${ }^{(15)}$. Além disso, um cuidador relacionou o risco de lesões em pacientes diabéticos com o uso de calçados inadequados, o que pode causar outras lesões, que não a LP e/ou LF.

Além das lesões causadas pela DM e a diminuição da cicatrização, os cuidadores apontaram também as lesões causadas por
nferm Atual In Derme v. 95, n. 34, 2021 e-021060 
varizes, sendo denominadas úlceras venosas (UV), que são lesões resultantes do retorno venoso inadequado e estão relacionadas à insuficiência venosa crônica, anomalias valvulares venosas e trombose venosa. As UV são mais comuns nos idosos, porém constatou-se que os pacientes que faziam uso de terapia compressiva (meia compressiva) e receberam orientação para elevação dos membros inferiores, apresentaram menor intensidade de dor e menor impacto nas atividades diárias ${ }^{(16)}$.

O último aspecto que emrgiu na primeira categoria temática diz respeito às $L F$, comumente encontrada em idosos. Os cuidadores relatam enquanto fatores de risco: bater em móveis, arrastar pernas sobre superfícies ásperas/pontiagudas ou rígidas, presença de dobras de lençóis e varizes. Referem-se à redução e eliminação destes riscos mediante a obtenção de um ambiente acessível ao idoso. Nessa perspectiva, cabe salientar que as LF podem ser provocadas por pequenos traumas, o que sinaliza para o enfermeiro, a necessidade de realizar ações educativas com pacientes, cuidadores e seus familiares sobre os riscos de um ambiente de cuidado desfavorável, técnicas corretas de manipulação, mudança de decúbito e transferência do paciente de um leito/maca para o outro para evitar fricções, cisalhamentos e contusões. Além disto, um programa dietético balanceado pode conferir maior resistência à pele. $\mathrm{O}$ plano de cuidados ao paciente com LF precisa ser elaborado de modo compartilhado, para que enfermeiros, demais integrantes da equipe, cuidadores, familiares e os próprios pacientes participem e https://doi.org/10.31011/reaid-2021-v.95-n.34-art.998 Rev Enferm Atual In Derme v. 95, n. 34, 2021 e-021060 contribuam para a definição do melhor caminho rumo ao sucesso terapêutico ${ }^{(17)}$.

Para além dos cuidados preventivos, os cuidadores colocam sobre suas ações de cuidados quando a lesão já está instalada, seja ela LP ou ST, o que configura a segunda categoria intitulada: Os cuidados com lesões na pele do idoso.

Nessa perspectiva os achados desta pesquisa são corroborados por outros estudos que trazem que a alimentação e hidratação em casos de lesão de pele são essenciais, uma vez que durante o tratamento das lesões destas, o corpo precisa de macro nutrientes, principalmente o carboidrato na forma de glicose, pois é este nutriente impede a degradação de proteínas essenciais em outras funções corporais, como também da água ${ }^{(18)}$.

Sobre o tratamento das lesões destacase a limpeza com solução fisiológica à $0,9 \%$, o que é amplamente consolidado na literatura vigente atual. Sobre as terapêuticas empregadas destacaram: uso de AGE, de placa de hidrocolóide e de colagenase. A solução fisiológica $0,9 \%$ possui grande disseminação na limpeza de feridas fechadas e abertas, principalmente por ser isotônica, com propriedades que não agridem o tecido da ferida. A SF pode ser utilizada na limpeza mecânica, através do jato de soro, e na aplicação simples da solução sem o jato na ferida, sendo que em feridas abertas com cicatrização por segunda e terceira intenção com presença de tecidos desvitalizados recomenda-se o uso de jato de soro ${ }^{(19)}$. 
Os Ácidos Graxos Essenciais (AGE) são compostos exclusivamente pelo ácido linoleico e o ácido linolênico. Ambos são considerados essenciais para o ser humano, principalmente devido a dois fatores: não são sintetizados pelo organismo e a ausência de sua ingestão pode gerar alterações dérmicas. Atualmente são utilizados produtos à base de AGE, vitamina A e E, além de lecitina de soja, tanto para o tratamento quanto para a prevenção de lesões, principalmente a $\operatorname{LP}^{(12)}$.

Sobre a placa de hidrocolóide, geralmente esta é recomendada no tratamento de LP de estágio II e III, com profundidade mínima. O tratamento com placa de hidrocolóide aumenta significativamente a taxa de cicatrização de $\operatorname{LP}^{(12)}$. Já a colagenase é uma cobertura enzimática que tem por substrato o colágeno nativo e desnaturalizado, cuja função é remover os restos celulares do tecido necrosado, ou seja, realizar o desbridamento químico, estimulando indiretamente a formação do tecido de granulação e posteriormente a reepitelização.

A colagenase é uma das substâncias mais utilizadas para o tratamento de feridas, incluindo as LP, sendo rotineiramente utilizada por profissionais em busca do progresso da lesão. No entanto, precisa ser utilizada adequadamente, com critérios específicos para sua indicação, periodicidade de troca, pois seu inadequado emprego pode ocasionar retardo cicatricial $^{(20)}$.

No que tange à prevenção e ao cuidado de pessoas com lesões de pele, a Resolução COFEN 567/2018 regulamenta as atribuições da equipe de Enfermagem no cuidado à ferida. Assim sendo, cabe ao enfermeiro realizar curativos, coordenar e supervisionar a equipe de enfermagem na prevenção e cuidados de feridas, dentre outras atribuições específicas. Ao técnico de enfermagem compete realizar curativos nas feridas de Estágio I e II, em estágio III somente quando delegado pelo enfermeiro, auxiliar o enfermeiro nos curativos de feridas de estágio III e IV, executar as ações prescritas pelo enfermeiro, dentre outras ${ }^{(20)}$.

Por fim, a enfermagem vem, essencialmente, no exercício profissional o cuidado ao indivíduo, família e coletividades do território, desempenhando as atividades com foco na promoção, prevenção, recuperação e reabilitação da saúde e, portanto, uma atuação fundamental para $o$ fortalecimento da Atenção Primária. Dentre tantos aspectos, destaca-se a demanda por cuidados à pessoa com lesão de pele, problema que tem sido motivo de discussão em diferentes esferas das práticas de saúde, especialmente no âmbito da gestão em saúde, uma vez que este tipo de lesão apresenta morbidade significativa, possui caráter reincidivante, contribui para a redução da qualidade de vida e eleva os gastos públicos com a saúde ${ }^{(20)}$.

\section{CONCLUSÃO}

A partir do estudo foi possível identificar os principais cuidados e ações desempenhados pelos cuidadores/familiares, sendo que a maioria desempenha esses cuidados de forma embasada pela literatura, no entanto boa parte delega este cuidado ao idoso. https://doi.org/10.31011/reaid-2021-v.95-n.34-art.998 Rev Enferm Atual In Derme v. 95, n. 34, 2021 e-021060 
Como enfermeiros, devemos realizar promoção da saúde, outra deficiência observada nos relatos dos entrevistados, pois ainda temos muito enraizado o tratamento de lesões e não a prevenção destas lesões.

Há uma grande falta de estudos sobre o papel do cuidador e do familiar no processo de prevenção e tratamento destas lesões, entretanto eles são fundamentais, como o enfermeiro, com estudos que corroborassem esse importância veríamos melhores meios de prevenir essas lesões e quais as áreas que os familiares tem maior deficiência, assim, melhorando o cuidado prestado tanto aos idosos quanto a família.

\section{REFERÊNCIAS}

1.Veras RP, Oliveira M. Aging in Brazil: the building of a healthcare model. Science \& Collective Health [Internet]. 2018 [acesso 2020 Abr 22];23(6):1929-1936. Disponível em:

https://www.scielosp.org/article/csc/2018.v23n 6/1929-1936/pt/

2.Santos-Orlandi AA, Brito TRP, Ottaviani AC, Rossetti ES, Zazzetta MS, Gratão ACM, Orlandi FS, Pavarini SCI. Perfil de idosos que cuidam de outros idosos em contexto de alta vulnerabilidade social. Escola Anna Nery [Internet]. 2017 [acesso 2020 Abr 22];21(1):e20170013. Disponível em: https://doi.org/10.5935/1414-8145.20170013 3.Tavares APC, Chaves Sá SP, Oliveira BG, Sousa AI. Qualidade de vida de idosos com úlceras de perna. Esc Anna Nery [Internet]. 2017 [acesso 2020 Abr 22];21(4):e20170134.
Disponível

em: http://www.scielo.br/pdf/ean/v21n4/pt_14148145-ean-2177-9465-EAN-2017-0134.pdf 4.Santos CT, Almeida MA, Lucena AF. Diagnóstico de enfermagem risco de úlcera por pressão: validação de conteúdo. Rev. LatinoAm. Enfermagem [Internet]. 2016 [acesso 2020 Abr 22];24:e2693. Disponível em: http://www.scielo.br/pdf/rlae/v24/pt_01041169-rlae-24-02693.pdf

5.Strazzieri-Pulido KC, Peres GRP, Campanili TC, Santos VLC. Prevalência de lesão por fricção e fatores associados: revisão sistemática. Rev. esc. enferm. USP [Internet]. 2015 [acesso 2020 Abr 22];49(4). Disponível em: http://www.scielo.br/scielo.php?pid=S008062342015000400674\&script=sci_arttext\&tlng $=\mathrm{pt}$

6.Prefeitura de Florianópolis. Disponível em: http://pmf.sc.gov.br/entidades/sa\%C3\%BAde/i ndex.php

7.Cândido KP, De Souza JC, De Oliveira FM. Perfil das pessoas com lesão por pressão na reabilitação: relação entre braden e dependência funcional. Revista Enfermagem Atual InDerme [Internet]. 2019 [acesso 2020 abr 22];87(Edição Esp, 2019). Disponível em: https://revistaenfermagematual.com.br/index.p $\mathrm{hp} /$ revista/article/view/164

8.Vieira VAS. Risco de lesão por pressão em idosos com comprometimento na realização de atividades diárias. Rev. De Enfermagem do Centro-Oeste Mineiro[Internet]. 2018 [acesso 2020 Abr 22];8:e2599. Disponível em: http://www.seer.ufsj.edu.br/index.php/recom/a https://doi.org/10.31011/reaid-2021-v.95-n.34-art.998 Rev Enferm Atual In Derme v. 95, n. 34, 2021 e-021060 
rticle/view/2599/1940

9.Facchinetti JB, Fernandes FP. Recursos utilizados por Fisioterapeutas para Prevenção e Tratamento de Lesão por Pressão. Id on Line Revista Multidisciplinar e de Psicologia [Internet]. 2017 [acesso 2020 Abr22];11(37). Disponível

em: https://idonline.emnuvens.com.br/id/article/vie w/811/1203

10.Vasconcelos JMB, Caliri MHL. Ações de enfermagem antes e após um protocolo de prevenção de lesões por pressão em terapia intensiva. Esc. Anna Nery, [Internet]. 2017[acesso 2020 Abr 22];21(1):e20170001.

Disponível em: http://www.scielo.br/pdf/ean/v21n1/14148145-ean-21-01-e20170001.pdf

11.Martins ESR. et al. Efeitos da ação dos ácidos graxos na pele sadia por biometria cutânea. Revista Enfermagem Atual InDerme [Internet]. 2017 [acesso 2020 Abr 22];82(20). Disponível em: https://pesquisa.bvsalud.org/portal/resource/pt/ biblio-1024015

12.Donoso MTV, et al. Análise de custos do tratamento de lesão por pressão em pacientes internados. Revista de Enfermagem do CentroOeste Mineiro [Internet]. 2019 [acesso 2020 Abr 22];9:e3446. Disponível em: http://seer.ufsj.edu.br/index.php/recom/article/ view/3446

13.De Sales DO, Waters C. O uso da Escala de Braden para prevenção de lesão por pressão em pacientes internados em Unidade de Terapia Intensiva/The use of the Braden Scale to prevent pressure injury in intensive care unit patients. Brazilian Journal of Health Review [Internet]. 2019 [acesso 2020 Abr 22];2(6):4900-492. Disponível em: http://www.brazilianjournals.com/index.php/B JHR/article/view/4300

14.Garbaccio JL, Ferreira AD, Pereira ALGG. Conhecimento e prática referidos por idosos no autocuidado com a pele no Centro-Oeste de Minas Gerais. Revista Brasileira de Geriatria e Gerontologia [Internet]. 2016 [acesso 2020 Abr 22];19(1):45-66. Disponível em: https://www.redalyc.org/pdf/4038/4038447730 05.pdf

15.Macedo JL. Eficácia da fitoterapia no processo de cicatrização tecidual de pacientes com diagnóstico de diabetes mellitus. Rev. Ciências e Saberes [Internet]. 2017 [acesso 2020 Abr 22];3(1):396-400. Disponível em: http://facema.edu.br/ojs/index.php/ReOnFace ma/article/view/182/85

16.Dantas DV. Validação clínica de protocolo para úlceras venosas na alta complexidade. Rev. Gaúcha de Enfermagem[Internet]. 2016 [acesso 2020 Abr 22];37(4):e59502. Disponível em: http://www.scielo.br/pdf/rgenf/v37n4/01026933-rgenf-1983-144720160459502.pdf 17.Tristão FR, et al. Práticas de Cuidados do Enfermeiro na Atenção Primária à Saúde: Gestão do cuidado da pele do idoso. Cogitare Enfermagem [Internet]. 2020 [acesso $2020 \mathrm{Abr}$ 22];25: e65223. Dísponível em: https://revistas.ufpr.br/cogitare/article/view/65 223

18.Dantas EMA. Tratamento e prevenção de lesão por pressão através de terapia https://doi.org/10.31011/reaid-2021-v.95-n.34-art.998 Rev Enferm Atual In Derme v. 95, n. 34, 2021 e-021060 
nutricional. International Journal Of Nutrology [Internet]. 2018 [acesso 2020 Abr 22];11(1). Disponível em: https://www.thiemeconnect.com/products/ejournals/abstract/10.1055/s $-0038-1675072$

19.Jesus BC, Ramos GF, Silva CCR, Gomes VCO, Silva GTR. Simulação em manequins como estratégia de ensino-aprendizagem para avaliação de ferida: relato de experiência. Rev. Estima [Internet]. 2017 [acesso 2020 Abr 22];15(4):245-249. Disponível em: https://www.researchgate.net/profile/Gilberto_ Tadeu_Silva/publication/322073510_Simulaca o_em_manequins_como_estrategia_de_ensino aprendizagem_para_avaliacao_de_ferida_relat o_de_experiencia/links/5a5395c40f7e9bbc105 6fbd9/Simulacao-em-manequins-comoestrategia-de-ensino-aprendizagem-paraavaliacao-de-ferida-relato-de-experiencia.pdf 20. Torres SB, de Queiroz ALFG, dos Santos ANA, Alves GQ, da Silva IA, Brito JKC. Óleo de girassol (Helianthus annus L.) como cicatrizante de feridas em idosos diabéticos. Brazilian Journal of Health Review [Internet]. 2021 [acesso 2021 Abr 15];4(2):4692-4703. Disponível em: https://www.brazilianjournals.com/index.php/ BJHR/article/view/25858

Submissão: 2021-01-12

Aprovado: 2021-04-16 